\title{
The Impact of a Single Administration of Intranasal Oxytocin on the Recognition of Basic Emotions in Humans: A Meta-Analysis
}

\author{
Sara Shahrestani ', Andrew H Kemp*,2 and Adam J Guastella*,' \\ 'Brain and Mind Research Institute, The University of Sydney, Camperdown, NSW, Australia; ${ }^{2}$ SCAN Research and Teaching Unit, School of \\ Psychology, The University of Sydney, Sydney, NSW, Australia
}

\begin{abstract}
Many studies have highlighted the potential of oxytocin (OT) to enhance facial affect recognition in healthy humans. However, inconsistencies have emerged with regard to the influence of OT on the recognition of specific emotional expressions (happy, angry, fear, surprise, disgust, and sadness). In this study, we conducted a meta-analysis of seven studies comprising 38I research participants (7I females) examining responses to the basic emotion types to assess whether OT enhances the recognition of emotion from human faces and whether this was influenced by the emotion expression and exposure time of the face. Results showed that intranasal OT administration enhances emotion recognition of faces overall, with a Hedges g effect size of 0.29 . When analysis was restricted to facial expression types, significant effects of OT on recognition accuracy were specifically found for the recognition of happy and fear faces. We also found that effect sizes increased to moderate when exposure time of the photograph was restricted to early phase recognition ( $<300 \mathrm{~ms}$ ) for happy and angry faces, or later phase recognition for fear faces (>300 ms). The results of the meta-analysis further suggest that OT has potential as a treatment to improve the recognition of emotion in faces, allowing individuals to improve their insight into the intentions, desires, and mental states of others.

Neuropsychopharmacology (2013) 38, 1929-1936; doi:I0.1038/npp.2013.86; published online 5 June 2013
\end{abstract}

Keywords: emotion; oxytocin; perception; face; expression, peptide

\section{INTRODUCTION}

Emotion recognition is a universal and central component of social cognition that provides the capacity to understand the intentions, feelings, and reactions of others (Elfenbein and Ambady, 2002). The initial experiments of Ekman et al (1969) revealed the capacity for even the most isolated tribes of New Guinea to understand the basic emotions of Western faces. Proficiency in recognizing emotions has been linked to altruistic helping behavior (Marsh et al, 2007), higher relationship quality, and a lowered rate of depression (Carton et al, 1999). Deficits in emotion recognition have also been associated with increased social anxiety, social avoidance, distress, depression, antisocial behaviors, and psychopathy (Mcclure and Nowicki, 2001). There is also growing awareness that social cognition is a unique and important neurocognitive domain for human

*Correspondence: Dr AH Kemp, SCAN Research and Teaching Unit, School of Psychology, Brennan MacCallum Building (A I8), The University of Sydney, Sydney, NSW 2006, Australia, Tel: +61 2 93517585, Fax: + 6129036 5223, E-mail: andrew.kemp@sydney.edu.au

Dr AJ Guastella, Brain and Mind Research Institute, The University of Sydney, 100 Mallet Street, Camperdown, NSW 2050, Australia, Tel: +612935। 0539, Fax: + 61293510855 ,

E-mail: adam.guastella@sydney.edu.au

Received 24 January 20 I3; revised II March 2013; accepted 16 March 2013; accepted article preview online 10 April 2013 social function (Shultz and Dunbar, 2012). Social cognitive deficits, including emotion recognition, predict social function in both early psychosis and autism above other tests of neurocognition (Bertrand et al, 2007; Losh et al., 2009). Thus, enhancement of the ability to recognize emotion in faces has the potential to improve social functioning in healthy humans and across clinical disorders.

Oxytocin (OT), a neuropeptide secreted from the posterior pituitary, has a critical role in mammalian social behavior (Macdonald and Macdonald, 2010). In healthy humans, the impact of OT on social cognition has been evaluated using a single dose of intranasal OT, typically given $30-45 \mathrm{~min}$ before the experimental task (see Guastella and Macleod (2012) for a review). Although many studies report that OT improves the perception of happy faces (Marsh et al, 2010; Schulze et al, 2011), others report that OT improves the recognition of angry, sad, or fear emotions in faces (Fischer-Shofty et al, 2010; Ellenbogen et al, 2012; Lischke et al, 2012). We have previously argued that different methodologies, or samples assessed may contribute to such inconsistent findings (Guastella and Macleod, 2012). For example, recognition performance for faces that are easier to read, such as happy faces (Hess et al, 1997), may be at ceiling levels in experiments that use longer exposure intervals (FischerShofty et al, 2010). 
A recent meta-analysis performed by Van IJzendoorn and Bakermans-Kranenburg (2012) revealed that OT significantly increased individual's response to facial expressions of emotion and in-group trust. However, their focus was very broad; their meta-analysis included studies on neuroanatomical measures, such as amygdala activation (eg, Domes et al, 2007), measures of trustworthiness and attractiveness (Theodoridou et al, 2009), and behavioral key presses in response to online social contingency (eg, Alvares et al, 2010). Outcomes from these studies were combined with more traditional assessments of facial recognition accuracy to produce the overall effect size of $d=0.21$. This study also did not investigate whether OT differentially impacted the ability to detect specific expressions in faces or other aspects of the experimental design. Thus, the overall effect of OT on the capacity to recognize emotion in faces remains unclear and further research is required to understand factors that may moderate any effect.

The present meta-analysis focuses only on recognition accuracy of full-face, basic expressions of emotion. Here, we focus on studies that recruited healthy controls, and excluded studies on patient populations to avoid biasing results as many clinical conditions display specific deficits in emotion recognition (Marsh and Blair, 2008). Our aim was to determine whether OT improved the accuracy of emotion recognition from faces, and whether this effect was influenced by emotion expression of the face stimuli (happy, sad, angry, fearful, surprised, and disgusted) and exposure time (short $<300 \mathrm{~ms}$; long $>300 \mathrm{~ms}$ ). Our central hypothesis was that OT would enhance the recognition of emotions overall. We further expected that emotion expressions that are easier to read, such as happy and angry faces, would show effects of OT enhancement at earlier rather than later exposure intervals, where ceiling effects may be more common (Hess et al, 1997; Leppänen and Hietanen, 2004; Fox et al, 2000).

\section{MATERIALS AND METHODS}

\section{Criteria for Considering Studies for this Review}

All randomized controlled trials of intranasal OT administration $v$ s placebo were included (study $n=7$ ). All studies examined perception or response in the form of overt choices in the recognition accuracy of both positive and negative emotional stimulus. This allowed us to compare dependent measures across categories of different expression types.

\section{Inclusion and Exclusion Criteria}

Participants included in this review were healthy controls. While many studies have examined individuals with psychopathology, such as autism, schizophrenia, and frontotemporal dementia (Guastella et al., 2010; Goldman et al, 2011; Jesso et al., 2011), the confounding effects of psychopathology were avoided in this study to draw more specific conclusions on the impact of OT on the recognition of differential expressions of facial emotion in healthy controls. Further, papers that used photographs of regions of the face rather than full-face images were not included to avoid confounding results with region presented (eg, Fischer-Shofty et al, 2010).

\section{Types of Intervention}

All doses of OT were administered intranasally; the most common dosage was 24 International Units (IU), with six of the seven studies administering OT at this dosage, and one study administering $40 \mathrm{IU}$ (Leknes et al, 2012). Twenty-four International Units is the most common dosage because of historical reasons; however, it is unclear whether other more optimal doses exist or whether someone who does not respond to lower doses will respond to higher ones (see Guastella et al, 2013) with respect to the latest recommendations relating to nasal spray administration).

\section{Primary Outcome Measures}

Observable behavioral responses such as recognition accuracy or reaction time are the only scientifically acceptable method of evaluating the veracity of cognitive models (Guastella and Macleod, 2012). In this regard, recognition accuracy was chosen as the primary outcome measure for the present meta-analysis; there was insufficient data available to conduct an analysis on reaction time data. Further, we chose not to combine these different dependent measures as speed of responding will confound accuracy of responding, leading to less meaningful results. Statistics pertaining to intensity thresholds for recognition were also avoided to prevent confounding results.

Recognition accuracy involves identifying specific facial expressions of emotion such as happy, angry, sad, and so on (Marsh et al, 2010; Schulze et al, 2011). Only full-faced photographs from validated databases of emotional expressions were included in the meta-analysis to standardize the task. Satisfactory reporting of statistics (eg, mean, SD, $p$-, $t$-, $r$-, or F-value, etc) in text or in tables was required for inclusion of a study. The threshold between conscious and nonconscious perception was set at $300 \mathrm{~ms}$ on the basis of previous literature (Del Cul et al, 2007).

\section{Search Methods for Identification of Studies}

Initial search. The search strategy followed guidelines outlined in the Preferred Reporting Items for Systematic Reviews and Meta-Analyses (PRISMA) (Moher et al, 2009), previously the QUality Of Reporting Of Meta-analyses (QUORUM) (Moher et al, 2000) statement.

Electronic databases including PsycINFO, PubMed/MEDLINE, and EMBASE were searched using combinations of the following: oxytocin, emotion, affect, face, and facial as per earlier meta-analyses focusing on different, although related research questions (Marsh and Blair, 2008). Databases were searched using the following terms: ('emotions'[MeSH Terms] OR 'emotions'[All Fields] OR 'emotion'[All Fields]) OR ('affect'[MeSH Terms] OR 'affect' [All Fields])) AND ('oxytocin'[MeSH Terms] OR 'oxytocin' [All Fields]) (('face'[MeSH Terms] OR 'face'[All Fields]) OR ('face'[MeSH Terms] OR 'face'[All Fields] OR 'facial'[All Fields])) AND ('oxytocin'[MeSH Terms] OR 'oxytocin'[All Fields]). In addition to these electronic searches, each report's citation list was examined for additional studies. 
Reference lists of relevant review papers identified in the literature search were also examined.

Data collection. The search strategy involved screening titles and abstracts for duplicates and identifying ineligible studies. Using full copies of the papers, two researchers (SS and JK) independently assessed whether studies met the inclusion criteria, and disagreements were resolved through discussion. Relevant statistics were then extracted from the eligible studies and included in the meta-analysis.

Aspects relating to study quality were assessed, including the extent of blinding, whether groups were randomized and whether the number of participants in the placebo and control conditions was comparable. This information is presented in a table describing features of the included studies (see Table 1).

Study Characteristics. Seven articles met our inclusion criteria from a total of 2683 studies (see Figure 1 outlining the number of papers included and excluded at each stage of the meta-analysis). These included seven independent studies comprising 381 research participants (71 females). The average number of participants per study was 54; two studies used The Pictures of Facial Affect Database (Ekman and Friesen, 1976), three used images from the Karolinska Directed Emotional Faces (Calvo and Lundqvist, 2008) series, another used the Dynamic Affect Recognition Evaluation (DARE) from the Cohn-Kanade database, and the remaining study using another validated stimulus set, the FACES database containing facial expressions of younger, middle-aged, and older women and men (Ebner et al, 2010).

\section{Statistical Analyses}

The Comprehensive Meta-Analysis (Borenstein et al, 2005) program was used to transform results of individual studies into the common metric of Hedges $g$. Hedges $g$ is a measure of the standardized difference between intervention and control condition that corrects for biases associated with small sample sizes and can be interpreted in the same way as Cohen's $d ; 0.2$ represents a small effect, 0.5 a medium effect, and 0.8 a large effect.

Heterogeneity across studies was assessed using the Q-statistic (Borenstein et al, 2009). A significant Q-statistic indicates dissimilar effect sizes across studies, indicating potential differences in methodology or study population across studies.

To determine whether any publication bias was present, the funnel plot was inspected for asymmetry. This technique determines whether there was a significant risk of bias, and controls for that risk by imputing values to correct for the bias (Begg, 1994).

An initial meta-analysis on the effects of emotional photographs of faces, regardless of expression type, was run to determine an overall effect of OT on emotion recognition, followed by moderator analyses to determine whether OT has an impact on specific expressions. A second moderator analysis was conducted to determine whether OTs impact on the recognition of emotions was moderated by implicit or explicit presentation of stimuli. The threshold between conscious and nonconscious perception was set at $300 \mathrm{~ms}$ on the basis of previous literature (Del Cul et al, 2007).

\section{RESULTS}

OT facilitated emotion recognition regardless of facial expression type $(g=0.291, p<0.001,95 \%$ CI: 0.154, 0.429 ), an effect associated with a small effect size. Inspection of symmetry in the funnel plot of standard error by Hedges' $g$ revealed no evidence of a significant publication bias, nor was there any indication of study heterogeneity $(Q(\mathrm{df}=13)=15.361, p>0.05)$.

\section{Moderation by Emotion Type}

A moderator analysis was conducted on facial expression type to determine whether the OT differentially impacts on the recognition of specific facial expressions. OT facilitated the recognition of all facial expressions; however, the effects of happy and fearful faces displayed statistically significant effects relative to placebo, see Figure 2.

Meta-analysis revealed significant effects of OT on emotion recognition of happy faces $(n=5, g=0.290$, $p=0.019,95 \%$ CI: $0.047,0.533)$ and fearful faces $(n=1$, $g=0.591, p=0.044,95 \%$ CI: $0.016,1.166)$, while trend level effects of OT were observed for the recognition accuracy of angry $(n=5, g=0.210, p=0.059,95 \%$ CI: $-0.008,0.428)$ and sad ( $n=1, g=0.471, p=0.075,95 \%$ CI: $-0.048,0.991)$ facial expressions (see Table 2). The impact of OT on faces in which multiple expressions were presented and emotion type was not differentiated $(n=2, g=0.343, p=0.308,95 \%$ CI: $-0.316,1.002$ ) were not significant (see Table 1). Eighty-five percent CIs were overlapping and inspection of heterogeneity Q-statistic for pairwise comparisons of all facial expression categories was nonsignificant $(p>0.05)$.

\section{Moderation by Implicit or Explicit Presentation}

Moderator analyses revealed that emotion recognition in both implicit and explicit presentations were significant $(n=8, g=0.240, p=0.007,95 \%$ CI: $0.064,0.415$ and $n=5$, $g=0.459, p<0.001,95 \%$ CI: $0.227,0.640$, respectively). Furthermore, a significant Q-statistic indicated that these categories did not differ from one another $(p>0.05)$. When grouping each of the effect sizes by emotion and further categorizing as explicit or implicit recognition, the implicit recognition of anger $(n=2, g=0.435, p=0.002,95 \%$ CI: $0.160,0.710)$, happiness $(n=3, g=0.447, p=0.009,95 \%$ CI: $0.114,0.781)$, and combined emotions $(n=1, g=0.673$, $p=0.013,95 \%$ CI: $0.141,1.204)$ were significant along with the explicit recognition of fear $(n=1, g=0.591, p=0.044$, 95\% CI: $0.016,1.166$ ), see Table 3 . There were no statistics for the implicit presentation of fear or sadness, so we were not able to conduct these comparisons; however, aside from these, all unmentioned groupings and comparisons were nonsignificant $(p>0.05)$.

\section{DISCUSSION}

This study demonstrates that OT nasal spray enhances the human capacity to recognize basic facial expressions of 


\section{Meta-analysis: OT on recognition of basic emotions}

S Shahrestani et al

Table I Summary of Studies used in Meta-Analysis

\begin{tabular}{ll}
\hline Reference & Sample \\
\hline $\begin{array}{l}\text { Domes et al } \\
(2012 a)\end{array}$ & $\begin{array}{l}\text { Sixty-nine male participants (mean } \\
\text { age }=\text { SD: } 24.0 \pm 3.1 \text { years). Twenty-four } \\
\text { International Unit of oxytocin was } \\
\text { administered }\end{array}$ \\
\\
\\
$\begin{array}{ll}\text { Domes et al } \\
\text { (2012b) }\end{array}$ & $\begin{array}{l}\text { Sixty-two healthy male volunteers (mean } \\
\text { age }=\text { SD: } 24.0 \pm 2.5 \text { years) participated } \\
\text { and were administered } 24 I U \text { of oxytocin }\end{array}$
\end{tabular}

Ellenbogen et al

Leknes et al (2012)

Lischke et al (2012)

Marsh et al (2010)

Schulze et al (2011)
Final sample consisted of 57 participants ( 15 females and 13 males) administered oxytocin $(24 I U)$ and 29 participants administered placebo ( 15 females and 14 males). Exclusion criteria included smoking, consuming legal or illegal drugs, being not fluent in English, currently ill, or suffering from a chronic medical condition, major sensory impairment, or lifetime mental disorder

Forty healthy right-handed adults were recruited, of which 20 were female. Forty International Unit oxytocin was administered

In a double-blind, placebo-controlled between-subjects design, 47 healthy male adults (age: $M=26.09, S D=3.41$ ) were randomly assigned to receive a nasal spray containing $24 \mathrm{IU}$ of oxytocin or placebo

\section{Method}

Dot-probe task was used with angry, happy, and neutral faces from the Karolinska Directed Emotional Faces
database. A pair of angry/neutral, happy/ neutral, or neutral/neutral facia expressions was presented for $100 \mathrm{~ms}$ vs $500 \mathrm{~ms}$. In emotional trials the probe appeared at the location of the emotional (congruent) face and at the location of the neutral (incongruent) face

The original version of the Dynamic using the Cohn-Kanade database of facial expressions. Each trial begins with a neutral facial expression that slowly changed into one of two basic emotions (happiness and anger) over time. Tria duration ranged from 16 to $34 \mathrm{~s}$ and participants were asked to detect the emotion of the particular face presented as soon as possible

An adapted stimulus detection task was utilized in which subjects fixated on a centrally placed gray ' + ' sign on a black background. Subjects responded with a single key press as fast as possible when the target (a black dot) appeared in a rectangle on either the left or right. Before the target appeared, a cue picture of a sad, angry, or neutral face from the pictures of facial affect database, signaling the likely location of the target appeared in one of the rectangles. On engagement trials, the cue appeared in the same rectangle as the target. Cue pictures were presented for 750,200 , or $17 \mathrm{~ms}$ followed by a masking stimulus. Images were from the Pictures of Facial Affect Database

Participants viewed black and white images of faces displaying explicitly angry faces, implicitly angry faces, neutral faces, implicitly happy faces, and explicitly happy faces. While participants viewed images they received concomitant tactile stimulation; however, only results from visual stimuli were presented in this paper. Perceived mood or facial expression of stimuli was recorded on a binary scale (How happy was the person? Anchors: Not happy-happy and how angry was the person? Anchors: Not angry-angry). Images were from the Karolinska Directed Emotional Faces

Computer-manipulated images of faces whose neutral expression was gradually changed into an emotional and consisted of neutral, sad, angry, fearful, or happy expressions. Images were from the FACES database Affect Recognition Evaluation (DARE)

Oxytocin did not improve overall emotion recognition accuracy and no main effect of group was found for either happiness or ange

Engagement scores consisted of the neutral minus the emotional picture for sad and angry faces presented for $750 \mathrm{~ms}$. One-way ANOVA revealed a significant difference between oxytocin and placebo for valid trials with sad faces but not angry faces

Separate ANOVAs confirmed that oxytocin increased ratings of congruent and decreased ratings of incongruent emotional expression, sharpening the perception of emotion difference between this and the accuracy that would be expected by chance is set was use

Participants were exposed to an angry, happy, or neutral face was presented fo 18,35 , or $53 \mathrm{~ms}$, followed by a "mask' showing a neutral face. Participants were explicitly informed that two facial stimuli would always appear in each trial, although they might only perceive one. Before each block, an instruction was given regarding the target emotion in the following trials. Following each targetmask pair participants had 3 s to indicate mask pair, panticipow $3 \mathrm{~s}$ to indicate whether the target emotion was present or absent (four blocks angry present/ absent and four blocks happy present absent). Images were taken from the Karolinska Directed Emotional Faces
Was the task too easy? Were participants given too much time to perform the task?
Will the cueing association necessarily be strong enough and will participants have a bias to responding to either side of the screen aside from the cueing. How would presenting images for $>750 \mathrm{~ms}$ have affected results compared to $750 \mathrm{~ms}$ ?
Could the touch or stroking stimulus have confounded results? Why wasn't there a control group for no tactile stimulation?
Oxytocin had no effect on participants' recognition accuracy, which was high for happy expressions, moderate for angry and fearful expressions, and low for sad expressions. Follow-up analysis revealed oxytocin improved specific recognition accuracy for fearful faces but not othe expressions

The only emotion for which oxytocin significantly affected recognition rates was happiness. Participants who received oxytocin recognized happiness more quickly $(M=964.1 \mathrm{~ms}, \mathrm{SD}=248)$ than participants who received placebo $(M=1096.1 \mathrm{~ms}, \mathrm{SD}=334 ; t(48)=1.58$ $p=0.12$ ). This was nonsignificant

Blood samples collected 45 min after administration of oxytocin and at the same time as emotion detection task Venipuncture could be perceived as an aversive stimulus, confounding results. Participants were asked to respond when they recognized an emotion but then given time to pick one of four possible emotions (happy, angry, sad, and fearful). This sample space is limited and could allow guessing of responses

Tasks were completed $35 \mathrm{~min}$ after substance administration; however, most papers will wait $50 \mathrm{~min}$. Participants viewed faces for only $500 \mathrm{~ms}$. Does this represent some level of early processing? Were participants making a conscious, informed selection?

Subjects administered oxytocin showe enhanced recognition memory. Effect of oxytocin on emotion recognition was more pronounced for happy than angry faces
Participants were given only $3 \mathrm{~s}$ to respond, is this enough or may pressure have affect their ability to focus? 


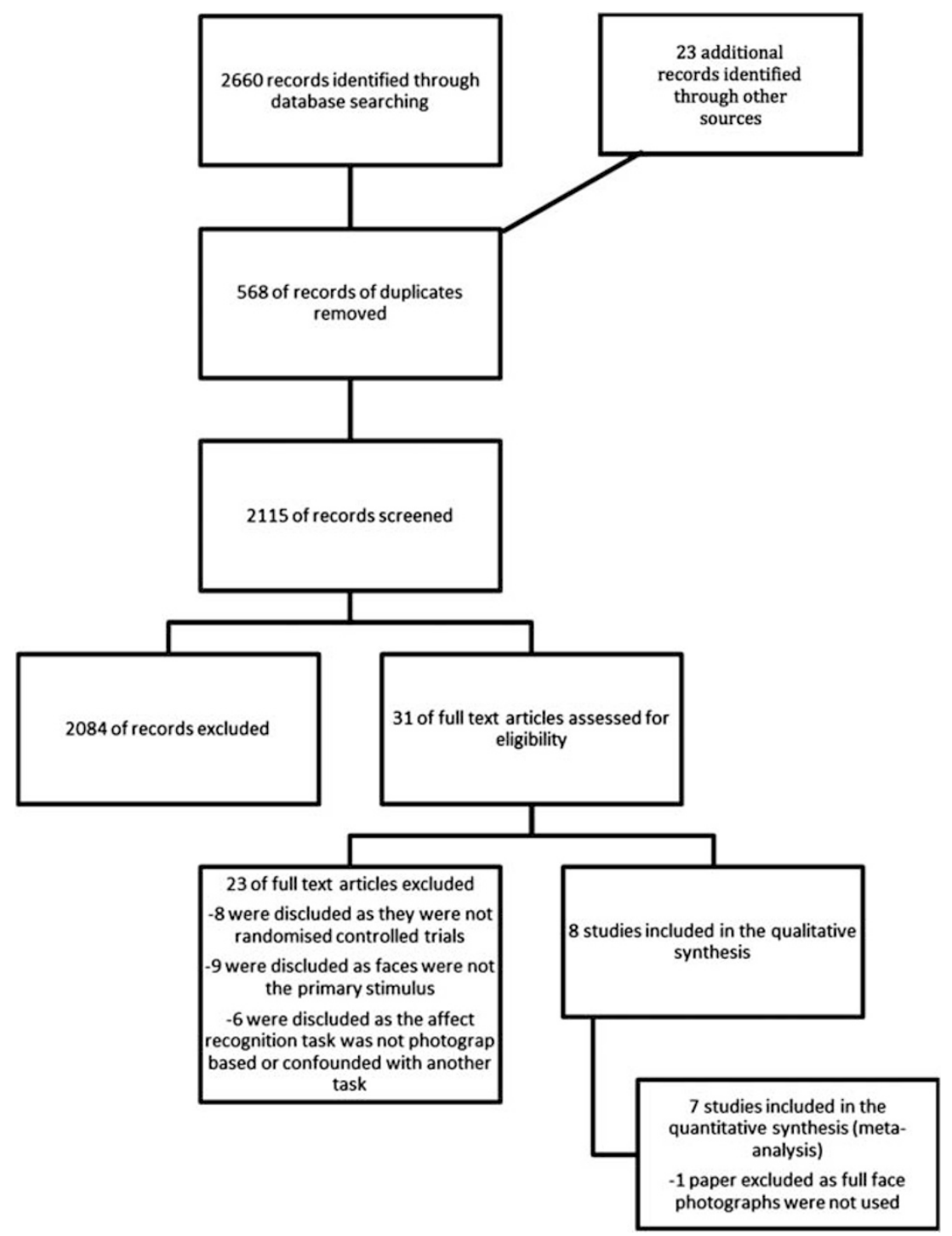

Figure I Summary of research process for meta-analysis, including numbers of papers included and excluded at each stage.

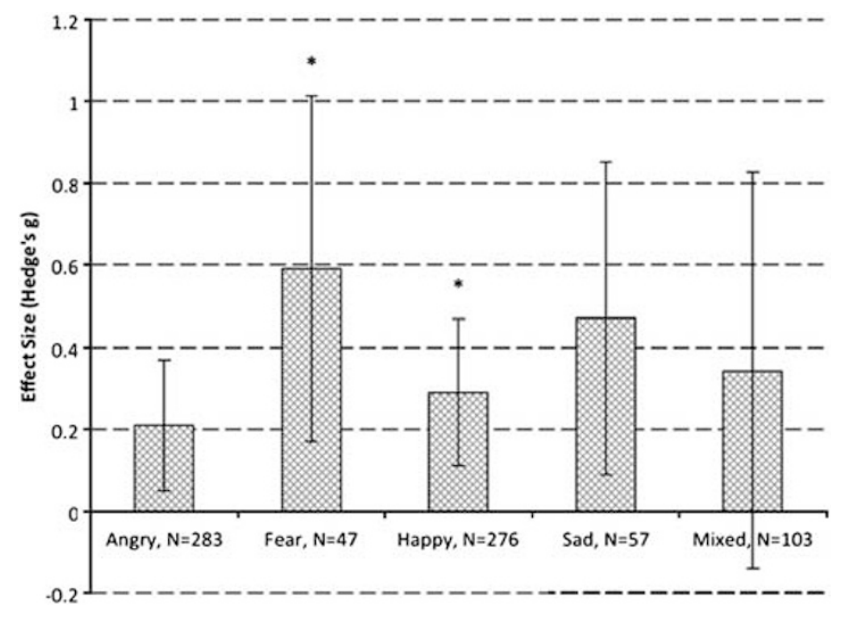

Figure 2 Effects of oxytocin on facial emotion recognition: combined effect sizes (g) and $85 \%$ confidence intervals. ${ }^{*} P<0.05$, indicating significance. emotion, an effect associated with small effect size $(g=0.291)$. Additional analyses of specific emotion expressions revealed that OT enhanced the recognition of happy and fearful facial expressions. The impact of OT on the recognition of angry and sad faces was at trend levels for the overall analyses. We then examined whether OT facilitated the recognition of face expressions under implicit $(<300 \mathrm{~ms})$ and explicit (>300 ms) presentation conditions (Del Cul et al, 2007). Results showed that under implicit recognition conditions, OT enhanced recognition of happy and angry expressions. Under longer durations of exposure, OT was found to enhance the recognition of fear expressions only.

The results of this study further support the view that OT nasal spray improves the facial recognition of emotion in humans. We found that OT enhances overall recognition accuracy, with effects for happy and fear faces specifically. The capacity for OT to improve emotion understanding, and the recognition of fear and happiness in particular, may 
Table 2 Moderator Analysis of Emotion Type on Recognition Accuracy Under Oxytocin

Recognition accuracy for different emotions

\begin{tabular}{lcclcc} 
Subgroup & No. of data sets & No. of participants under oxytocin & Effect size $\mathbf{( 9 5 \%} \mathbf{C l})$ & SE of summary effect size & Effect size $\boldsymbol{p}$-value \\
\hline Anger & 5 & 156 & $0.210(-0.008,0.428)$ & 0.111 & 0.059 \\
Fear & 1 & 24 & $0.591(0.016,1.166)$ & 0.293 & $0.044 * 3$ \\
Happy & 5 & 158 & $0.290(0.047,0.533)$ & $0.019 *$ & 0.336 \\
Mixed & 2 & 52 & $0.343(-0.316,1.002)$ & 0.265 \\
Sadness & I & 23 & $0.471(-0.048,0.991)$ & 0.075 \\
\hline
\end{tabular}

*P $P 0.05$, indicating significance.

Table 3 Moderator Analysis of Emotion Type and Implicit or Explicit Presentation on Recognition Accuracy Under Oxytocin

Recognition accuracy for different emotions

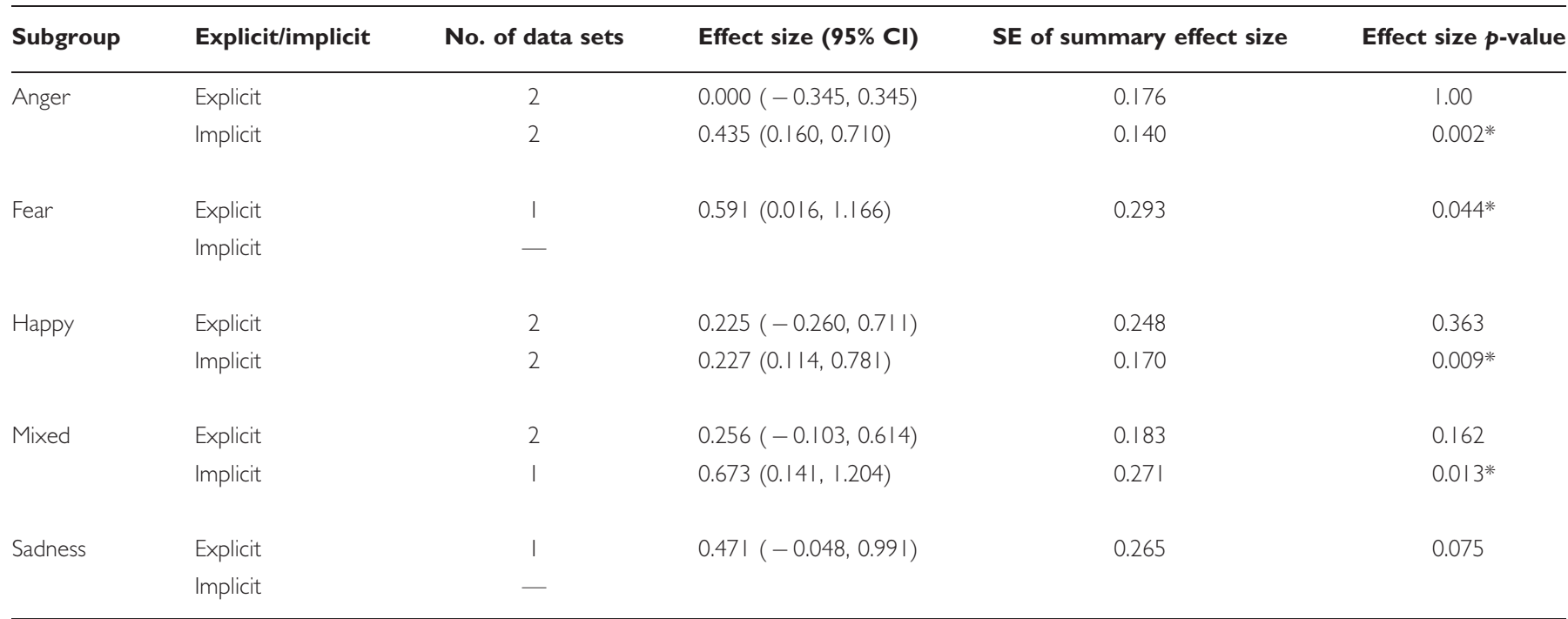

$* P<0.05$, indicating significance.

have important benefits to social engagement (Kemp and Guastella, 2011). Robust associations have been observed between antisocial behavior and impaired recognition of fearful expressions in particular (Marsh and Blair, 2008). It has been argued that the ability to recognize fear enables one to appreciate the pain and mental experience of others, and is therefore more likely to reduce antisocial tendencies (Marsh and Blair, 2008). This ability has also been linked experimentally to prosocial behavior (Marsh et al, 2007; Kirsch et al, 2005). Similarly, socially anxious individuals are more likely to interpret facial expressions in a negative manner contributing to both negative self-images and social avoidance (Garner et al, 2009; Guastella et al, 2009). Further research is now required to determine links between improvements in face recognition from OT and subsequent behavioral outcomes associated with reducing aggressive behavior and anxiety/avoidance in social situations. In light of recent evidence revealing that $\mathrm{OT}$ improves social cognition in individuals with frontotemporal dementia relative to baseline (Jesso et al, 2011), there may also be implications for the use of OT in disorders of neuropsychological deficit.

The findings from this study also suggest that the previously observed inconsistent results in this field may be partially associated with stimuli exposure duration. Happy and angry face expressions are more efficiently recognized in humans and require less time for processing (Hess et al, 1997; Leppänen and Hietanen, 2004; Fox et al, 2000). OT appears to further improve this fast recognition process in humans below the threshold of conscious awareness (Schulze et al, 2011). Implicit recognition is associated with the amygdalohippocampal junction, an important part of the limbic system (Critchley et al, 2000). Accumulating evidence points to OT as a particularly important hormone within this system (Insel and Young, 2000).

For facial expressions that may require more time to recognize, the facilitating effects of OT administration may be more likely at later stages of recognition processing. The present meta-analysis demonstrated significant effects on fear faces at the longer exposure duration. We note, however, that the weighted effect size obtained for fear was recovered from only one study. Further studies are therefore required across all of the basic emotions of facial expressions at short and long duration intervals to further gather data on this issue.

There is growing recognition for the need to develop robust markers of response to OT nasal spray, to determine when an individual is likely to respond favorably for 
longer-term therapeutic purposes (Guastella and Macleod, 2012). The results here support the view that enhanced capacity for face recognition, especially for happy or fearful faces, may provide a useful marker.

To further improve our understanding of the impact of OT on face recognition, we suggest the following directions for future research and recommendations for implementation:

First, we suggest that future studies need to assess emotion recognition across all basic emotion expressions. While studies have primarily focused on happy and angry faces as being representative of the positive and negative facial expressions, there is limited data on effects across all basic emotions. The field has now progressed beyond simple positive $v s$ negative single expression comparisons. Future research is required employing stimuli across the range of basic emotions of facial expression.

Second, research is needed to differentiate and assess both fast and longer exposure latencies. This meta-analysis suggests that exposure latencies of stimuli influence outcome. Timing (eg, pre-conscious, implicit presentations) of exposure needs to be incorporated into designs. Third, researchers need to use standardized full-faced photographs from established databases of emotion. Where the aim is to understand the effects on facial emotion recognition, preference could be given to full-faced photos given these stimuli are well validated for this purpose. Consideration also needs to be given to stimuli brightness and contrast, head size, hair cut and color, skin color and the presence of accessories (Gronenschild and Smeets, 2009). Fourth, more research is needed to assess mechanisms underlying emotion recognition enhancement of OT. Evaluation of the influence of OT on identity recognition (Calder and Young, 2005), independent of emotion decoding, is required. Studies could explore how OT influence holistic $v s$ featural processing Andari et al, 2010, recognition of expression intensity, or authentic $v s$ posed expressions. In addition, evaluation of emotion recognition in the context of other more traditional neuropsychological measures is required. Factors such as intelligence, attention, verbal ability, and task-specific motivation have all been associated with facial recognition performance (Marsh and Blair, 2008).

Finally, if these tests are to provide reliable markers of response to oxytocin administration, then systematic evaluation of dose and response to drug is required. Almost all of the studies testing emotion recognition in healthy samples have administered the standard dose of $24 \mathrm{IU}$. Systematic evaluation of how response changes according to dose and other measurable factors that influence bioavailability of nasal administration is required (Guastella et al, 2013). These changes should be evaluated in the context of a range of other variables thought to mark response to OT, such as heart rate variability (Kemp et al, 2012).

There are a number of limitations to the current metaanalysis. First, behavioral measures of rating each emotion differed across studies. Some studies used a multiple-choice format (Lischke et al, 2012; Marsh et al, 2010), while others used a cue-response format (Ellenbogen et al, 2012; Schulze et al, 2011), or ratings on a binary scale (Leknes et al, 2012). Second, while the meta-analysis weighted the effect size obtained for fear against other extracted effect sizes according to sample size, as discussed above, the effect size for fear was based on only one study. As such, further research must be conducted to support this obtained result.

Overall the results of this quantitative synthesis indicate that, from the current available data, the enhancement of emotion recognition by OT is driven by improved perception of fearful and happy facial expressions. Findings also point to the need for further research to corroborate findings in the emotions of disgust and surprise. The results of this quantitative synthesis suggest the potential of OT to be an effective treatment for deficits in the recognition of emotions. Specifically, patients with disorders such as schizophrenia, antisocial disorders, and social anxiety may receive some benefit from the improvement in processing of fear and happy emotions in faces following OT administration. Further, improving one's ability to understand emotion in another's face is likely to facilitate understanding of the intentions, desires, and mental states of others.

\section{ACKNOWLEDGEMENTS}

We thank Gail Alvares for comments on this manuscript and Jonathan Krygier for assisting with article preparation. This manuscript was partially supported by an ARC linkage project LP110100513 to Guastella. AHK is supported by a National Health and Medical Research Council (NHMRC) career development fellowship (571101).

\section{DISCLOSURE}

The authors declare no conflict of interest.

\section{REFERENCES}

Alvares GA, Hickie IB, Guastella AJ (2010). Acute effects of intranasal oxytocin on subjective and behavioral responses to social rejection. Exp Clin Psychopharmacol 18: 316-321.

Andari E, Duhamel JR, Zalla T, Herbrecht E, Leboyer M, Sirigu A (2010). Promoting social behavior with oxytocin in highfunctioning autism spectrum disorders. Proc Natl Acad Sci USA 107: 4389-4394.

Begg C (1994). Publication bias. The Handbook of Research Synthesis. Sage Publications.

Bertrand MC, Sutton H, Achim AM, Malla AK, Lepage M (2007). Social cognitive impairments in first episode psychosis. Schizophr Res 95: 124-133.

Borenstein M, Hedges L, Higgins J, Rothstein H (2005). Comprehensive Meta-analysis Version 2. Englewood, NJ: Biostat.

Borenstein M, Hedges LV, Higgins JPT, Rothstein HR (2009). Introduction to Meta-Analysis. Wiley: New York, NY, USA.

Calder AJ, Young AW (2005). Understanding the recognition of facial identity and facial expression. Nat Rev Neurosc 6: 641-652.

Calvo MG, Lundqvist D (2008). Facial expressions of emotion (KDEF): identification under different display-duration conditions. Behav Res Methods 40(1): 109-115.

Carton J, Kessler E, Pape C (1999). Nonverbal decoding skills and relationship well-being in adults. J Nonverbal Behav 23: 91-100.

Critchley H, Daly E, Phillips M, Brammer M, Bullmore E, Williams S et al (2000). Explicit and implicit neural mechanisms for processing of social information from facial expressions: a functional magnetic resonance imaging study. Hum Brain Mapp 9: 93-105. 
Del Cul A, Baillet S, Dehaene S (2007). Brain dynamics underlying the nonlinear threshold for access to consciousness. PLoS Bio 5: e260.

Domes G, Heinrichs M, Gläscher J, Büchel C, Braus DF, Herpertz SC (2007). Oxytocin attenuates amygdala responses to emotional faces regardless of valence. Biol Psychiatry 62: 1187-1190.

Domes G, Sibold M, Schulze L, Lischke A, Herpertz SC, Heinrichs M (2012a). Intranasal oxytocin increases covert attention to positive social cues. Psychol Med (e-pub ahead of print).

Domes G, Steiner A, Porges SW, Heinrichs M (2012b). Oxytocin differentially modulates eye gaze to naturalistic social signals of happiness and anger. Psychoneuroendocrinology 12: 00337-X.

Ebner NC, Riediger M, Lindenberger U (2010). FACES-a database of facial expressions in young, middle-aged, and older women and men: development and validation. Behav Res Methods 42: 351-362.

Ekman P, Friesen W (1976). The Pictures of Facial Affect Database. Consulting Psychologists Press: Palo Alto, CA, USA.

Ekman P, Sorenson ER, Friesen WV (1969). Pan-cultural elements in facial displays of emotion. Science (New York, NY) 164: 86-88.

Elfenbein HA, Ambady N (2002). On the universality and cultural specificity of emotion recognition: a meta-analysis. Psychol Bull 128: 203-235.

Ellenbogen MA, Linnen A, Grumet R, Cardoso C (2012). The acute effects of intranasal oxytocin on automatic and effortful attentional shifting to emotional faces. Psychophysiology 49: 128-137.

Fischer-Shofty M, Shamay-Tsoory SG, Harari H, Levkovitz Y (2010). The effect of intranasal administration of oxytocin on fear recognition. Neuropsychologia 48: 179-184.

Fox E, Lester V, Russo R, Bowles RJ, Pichler A, Dutton K (2000). Facial expressions of emotion: are angry faces detected more efficiently? Cogn Emot 14: 61-92.

Garner M, Baldwin DS, Bradley BP, Mogg K (2009). Impaired identification of fearful faces in generalised social phobia. J Affect Disord 115: 460-465.

Goldman MB, Gomes AM, Carter CS, Lee R (2011). Divergent effects of two different doses of intranasal oxytocin on facial affect discrimination in schizophrenic patients with and without polydipsia. Psychopharmacology 216: 101-110.

Gronenschild E, Smeets F (2009). The use of faces as stimuli in neuroimaging and psychological experiments: a procedure to standardize stimulus features. Behav Res Methods 41: 1053-1060.

Guastella AJ, Einfeld SL, Gray KM, Rinehart NJ, Tonge BJ, Lambert $\mathrm{TJ}$ et al (2010). Intranasal oxytocin improves emotion recognition for youth with autism spectrum disorders. Biol Psychiatry 67: 692-694.

Guastella AJ, Hickie IB, McGuinness MM, Otis M, Woods E, Disinger H (2013). Recommendations for the standardisation of oxytocin nasal administration and guidelines for its reporting in human research. Psychoneuroendocrinology 38: 612-625.

Guastella AJ, Howard AL, Dadds MR, Mitchell PB, Carson DS (2009). A randomized controlled trial of intranasal oxytocin as an adjunct to exposure therapy for Social Anxiety Disorder. Psychoneuroendocrinology 34: 917-923.

Guastella AJ, Macleod C (2012). A critical review of the influence of oxytocin nasal spray on social cognition in humans: evidence and future directions. Horm Behav 61: 773.

Hess U, Blairy S, Kleck RE (1997). The intensity of emotional facial expressions and decoding accuracy. J Nonverbal Behav 21: 241-257.

Insel T, Young L (2000). Neuropeptides and the evolution of social behavior. Curr Opin Neurobiol 10: 784-789.
Jesso S, Morlog D, Ross S, Pell MD, Pasternak SH, Mitchell DGV et al (2011). The effects of oxytocin on social cognition and behaviour in frontotemporal dementia. Brain 134(Part 9): 2493-2501.

Kemp AH, Quintana DS, Kuhnert RL, Griffiths K, Hickie IB, Guastella AJ (2012). Oxytocin increases heart rate variability in humans at rest: implications for social approach-related motivation and capacity for social engagement. PLoS One 7: e44014.

Kemp AH, Guastella AJ (2011). The role of oxytocin in human affect: a novel hypothesis. Curr Direct Psychol Sci 20: 222-231.

Kirsch P, Esslinger C, Chen Q, Mier D, Lis S, Siddhanti S et al (2005). Oxytocin modulates neural circuitry for social cognition and fear in humans. J Neurosci 25: 11489-11493.

Leknes S, Wessberg J, Ellingsen DM, Chelnokova O, Olausson H, Laeng B (2012). Oxytocin enhances pupil dilation and sensitivity to 'hidden' emotional expressions. Soc Cogn Affect Neurosci (e-pub ahead of print).

Leppänen JM, Hietanen JK (2004). Positive facial expressions are recognized faster than negative facial expressions, but why? Psychol Res 69: 22-29.

Lischke A., Berger C., Prehn K., Heinrichs M., Herpertz S. C., Domes G. (2012). Intranasal oxytocin enhances emotion recognition from dynamic facial expressions and leaves eyegaze unaffected. Psychoneuroendocrinology 37: 475-481.

Losh M, Adolphs R, Poe MD, Couture S, Penn D, Baranek GT et al (2009). Neuropsychological profile of autism and the broad autism phenotype. Archiv Gen Psychiatry 66: 518-26.

Macdonald K, Macdonald TM (2010). The peptide that binds: a systematic review of oxytocin and its prosocial effects in humans. Harvard Review Psychiatry 18: 1-21.

Marsh AA, Kozak MN, Ambady N (2007). Accurate identification of fear facial expressions predicts prosocial behavior. Emotion 7: 239-251.

Marsh AA, RJR Blair (2008). Deficits in facial affect recognition among antisocial populations: a meta-analysis. Neurosci Biobehav Rev 32: 454-465.

Marsh AA, Yu HH, Pine DS, Blair RJR (2010). Oxytocin improves specific recognition of positive facial expressions. Psychopharmacology 209: 225-332.

Mcclure EB, Nowicki S (2001). Associations between social anxiety and nonverbal processing skill in preadolescent boys and girls. J Nonverbal Behav 25: 3-19.

Moher D, Cook DJ, Eastwood S, Olkin I, Rennie D, Stroup DF (2000). Improving the quality of reports of meta-analyses of randomised controlled trials: The QUOROM Statement. Onkologie 23: 597-602.

Moher D, Liberati A, Tetzlaff J, Altman DG, Group TP (2009). Preferred reporting items for systematic reviews and metaanalyses: The PRISMA Statement. PLoS Clin Trials 6: e1000097.

Schulze L, Lischke A, Greif J, Herpertz SC, Heinrichs M, Domes G (2011). Oxytocin increases recognition of masked emotional faces. Psychoneuroendocrinology 36: 1378-1382.

Shultz S, Dunbar RI (2012). The social brain hypothesis: an evolutionary perspective on the neurobiology of social behavior. In: Richmond SRees GEdwards S (eds.) I Know What You're Thinking: Brain Imaging and Mental Privacy. Oxford University Press.

Theodoridou A, Rowe AC, Penton-Voak IS, Rogers PJ (2009). Oxytocin and social perception: oxytocin increases perceived facial trustworthiness and attractiveness. Horm Behav 56: 128-132.

Van IJzendoorn MH, Bakermans-Kranenburg MJ (2012). A sniff of trust: meta-analysis of the effects of intranasal oxytocin administration on face recognition, trust to in-group, and trust to out-group. Psychoneuroendocrinology 37: 438-443. 